\title{
Catalyst and Solvent-Free Microwave Assisted Expeditious Synthesis of 3-Indolyl-3-hydroxy Oxindoles and Unsymmetrical 3,3-Di(indolyl)indolin-2-ones
}

\author{
Prasanna K. Vuram1, C. Kabilan', Anju Chadha ${ }^{1,2^{*}}$ \\ ${ }^{1}$ Laboratory of Bioorganic Chemistry, Department of Biotechnology, Indian Institute of Technology Madras, \\ Chennai, India \\ ${ }^{2}$ National Centre for Catalysis Research, Indian Institute of Technology Madras, Chennai, India \\ Email: *anjuc@iitm.ac.in
}

Received 30 April 2015; accepted 27 June 2015; published 30 June 2015

Copyright (C) 2015 by authors and Scientific Research Publishing Inc.

This work is licensed under the Creative Commons Attribution International License (CC BY). http://creativecommons.org/licenses/by/4.0/

\section{(c) (i) Open Access}

\begin{abstract}
A simple and efficient method for the synthesis of 3-indolyl-3-hydroxy oxindoles and unsymmetrical 3,3-di(indolyl)indolin-2-ones using microwave irradiation without catalyst and solvent is described. A series of 3-indolyl-3-hydroxy oxindoles and unsymmetrical 3,3-di(indolyl)indolin-2ones have been synthesized in very short reaction times of 5 and 10 minutes and in yields ranging from $31 \%$ to $\mathbf{9 8 \%}$ and from $53 \%$ to $\mathbf{7 8 \%}$ respectively. This method offers a significant advantage over the conventional methods in terms of simplicity and shorter reaction time. To the best of our knowledge compounds $\mathrm{N}$-allyl-3-hydroxy-3-(1-methyl-indol-3-yl)indolin-2-one (6c), N-allyl-3hydroxy-3-(5-methoxy-indol-3-yl)indolin-2-one (8c), N-benzyl-3-hydroxy-3-(1-methyl-indol-3-yl) indolin-2-one (10c), N-propargyl-3-hydroxy-3-(1-methyl-indol-3-yl)indolin-2-one (13c), N-propargyl3-hydroxy-3-(5-methoxy-indol-3-yl)indolin-2-one (14c), 3-(5-methoxy-1H-indol-3-yl)-3-(1H-indol3-yl)indolin-2-one (1e), 3-1-methyl(5-methoxy-1H-indol-3-yl)-3-(1H-indol-3-yl)indolin-2-one (2e), 3-1-allyl(5-methoxy-1H-indol-3-yl)-3-(1H-indol-3-yl)indolin-2-one (3e), 3-1-benzyl(5-methoxy-1H-indol-3-yl)-3-(1H-indol-3-yl)indolin-2-one (4e) and 3-1-(prop-2-ynyl)(5-methoxy-1H-indol-3-yl)-3(1Hindol-3-yl)indolin-2-one (5e) are reported here for the first time. All the compounds are characterized by IR, ${ }^{1} \mathrm{H},{ }^{13} \mathrm{C}$ NMR and HRMS.
\end{abstract}

\footnotetext{
${ }^{*}$ Corresponding author.
}

How to cite this paper: Vuram, P.K., Kabilan, C. and Chadha, A. (2015) Catalyst and Solvent-Free Microwave Assisted Expeditious Synthesis of 3-Indolyl-3-hydroxy Oxindoles and Unsymmetrical 3,3-Di(indolyl)indolin-2-ones. International Journal of Organic Chemistry, 5, 108-118. http://dx.doi.org/10.4236/ijoc.2015.52012 


\section{Keywords}

\section{Catalyst-Free, Isatin, Indole, Microwave, Solvent-Free}

\section{Introduction}

Indoles and their derivative oxindoles are a privileged class of molecules in synthetic as well as biological chemistry. The hybrid molecules of indole and oxindole are also important due to their prevalent biological activities. For instance, the spermicidal activity of 3,3-bis(5-methoxy-1H-indol-3-yl)indolin-2-one and 3,3-bis(3-carboxymethyl-1H-indol-2-yl)indolin-2-one is higher than that of the standard spermicide "Nonoxynol-9" (N-9) [1]; di(indolyl)indolin-2-one derivatives showed strong and selective cytotoxicity against cancer cells [2]. These molecules are also known to possess antibacterial, anti-inflammatory and antiprotozoal activities [3]. In addition to these manifold biological applications, very recently 3-indolyl-3-hydroxy oxindoles (hybrid molecule of isatin and indole) were used as precursors in the synthesis of natural products (+)-gliocladin [4] and (+)-folicanthine [5] and in the synthesis of the heterocyclic analogue of BINAP [6]. The synthesis of 3-indolyl-3-hydroxy oxindoles involves a Friedel-Crafts type of electrophilic substitution between the electron-rich third position of the indole and electron deficient carbonyl group of the isatin [7]. This reaction usually results in the formation of symmetrical 3,3'-diindolyl oxindoles in a single step. Controlling the reaction at monosubstituted 3-indolyl-3-hydroxy oxindole stage is quite challenging.

The synthesis of symmetrical 3,3-di(indolyl)indolin-2-ones has been reported [8]-[22], while in the case of unsymmetrical 3,3-di(indolyl)indolin-2-ones, so far only three reports are available in the literature to the best of our knowledge. The synthesis involves a stepwise process. Wang and Ji reported an ultrasound irradiation method in the presence of ceric ammonium nitrate (CAN) for 1 to $5 \mathrm{~h}$ [23]; Moghadam and co-workers reported the reaction in the ionic-liquid N,N,N,N-tetramethylguanidinium trifluoroacetate (TMGT) for $1 \mathrm{~h}$ [24] and Nikpassand and co-workers reported it with a montmorillonite in 30 - 35 minutes [25].

In the case of 3-indolyl-3-hydroxy oxindoles, Kumar and co-workers reported the synthesis of various 3-indolyl-3-hydroxy oxindoles by supramolecular catalysis ( $\beta$-Cyclodextrin) in 45 - 190 minutes [7]; Shanthi and co-workers reported a time of 60 - 120 minutes using $\mathrm{K}_{2} \mathrm{CO}_{3}$ as a catalyst [26]; Meshram and co-workers reported a time of 15 minutes in the presence of Triton B [27]; Hosseini and Tavakolian reported a reaction time of $2.5 \mathrm{~h}$ using $\mathrm{ZnO}$ nanorods in aqueous medium [28]. EtOH and water medium (60:40) in the presence of Lewis acid $\left(\mathrm{FeCl}_{3} \cdot 6 \mathrm{H}_{2} \mathrm{O}\right)$ and ultrasonic irradiation in 5 - 25 minutes was reported by Khorshidi and Tabatabaeian [29] while Makarem and co-workers reported an electrochemical method using EtOH/Propanol (60 - 240 minutes) [30]. Srihari and Murthy reported a heterogeneous catalyst (Kaolin/KOH) in the presence of $\mathrm{MeOH}$ as a solvent in 138 - 470 minutes [31]. Using various ionic-liquids, Moghadam and co-workers reported a reaction time of 10 - 20 minutes [24]. Jing Deng and coworkers reported an enantioselective version by using cupreine [32]. Nadine and coworkers also reported the reaction using chiral scandium(III) and indium(III) pybox complexes [33]. Recently, Pravathaneni Sai Prathima and co-workers reported the synthesis in aqueous medium using a base catalyst-diethanolamine [34]. The focus of our lab is to develop "green" methods for organic synthesis. In addition to using biocatalysts for organic transformations, we have now used microwave irradiation for organic synthesis as reported in the present study. Use of solvent-free and catalyst-free reaction conditions is an attractive proposition as seen in numerous microwave-assisted reactions. To the best of our knowledge, this is the first report for catalyst and solvent-free synthesis of 3-indolyl-3-hydroxy oxindoles and unsymmetrical 3,3di(indolyl)indolin-2-ones under microwave irradiation in 5 and 10 minutes respectively.

\section{Results and Discussion}

Optimization of reaction conditions for the synthesis of 3-indolyl-3-hydroxy oxindoles, was carried out using a isatin (1a) [1 equivalent] and an indole (1b) [1.2 equivalents]. Using a microwave oven temperature at $100^{\circ} \mathrm{C}$ and irradiation for 5 minutes, gave only the symmetrical 3,3-di(indolyl)indolin-2-ones as product (Scheme 1). Decreasing the time of irradiation to 3 minutes and then to 1 minute also resulted in the formation of the symmetrical 3,3-di(indolyl)indolin-2-one product and in 1 minute, 3-hydroxy-3-(1H-indol-3-yl)indolin-2-one (1c) and unreacted starting materials isatin (1a) and indole (1b), were also detected. Then we envisioned that $100^{\circ} \mathrm{C}$ was not a suitable temperature for controlling the reaction at the mono substituted hydroxyl stage. The reaction was therefore carried out at reduced temperatures: at $90^{\circ} \mathrm{C}$ and $80^{\circ} \mathrm{C}$ for 5 minutes, a mixture of 3-hydroxy-3- 


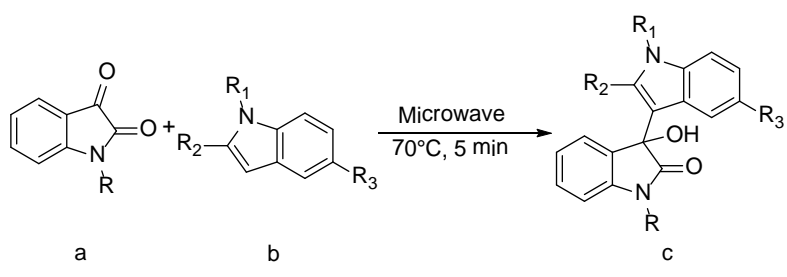

Scheme 1. Synthesis of various 3-indolyl-3-hydroxy oxindoles.

(1H-indol-3-yl)indolin-2-one (1c), symmetrical 3,3-di(indolyl)indolin-2-one in addition to unreacted starting material were observed. At $70^{\circ} \mathrm{C}$ for five minutes, only 3-hydroxy-3-(1H-indol-3-yl)indolin-2-one (1c) and unreacted starting materials were detected. But an increase in the reaction time from the sixth minute onwards, resulted in the formation of symmetrical 3,3-di(indolyl)indolin-2-one, even at $70^{\circ} \mathrm{C}$. Based on this observation, $70^{\circ} \mathrm{C}$ and 5 minutes were optimized as the reaction conditions for synthesizing 3-hydroxy-3-( $1 \mathrm{H}$-indol-3-yl) indolin-2-one (1c), but the isolated yield was only 31\%. Further increasing the amount of one of the starting materials i.e. three and five equivalents of indole (1b), showed no improvement in the yield of 3-hydroxy-3(1H-indol-3-yl)indolin-2-one (1c). Also with $\mathrm{N}$-allyl, and $\mathrm{N}$-benzyl isatins, the yields of N-allyl-3-hydroxy-3(1H-indol-3-yl)indolin-2-one (5c) and N-benzyl-3-hydroxy-3-(1H-indol-3-yl)indolin-2-one (9c) were only 34\% and $40 \%$ respectively. Based on the nucleophilicity index of the indole ring [35], N-methyl indole, 2-methyl indole and 5-methoxy indole were selected [these are electron-rich at the third position]. In addition, 5-nitro indole which is electron deficient at the third position was also selected. The reaction between 5-methoxy indole and simple isatin, gave a yield of $64 \%$ for 5-Methoxy-3-hydroxy-3-(1H-indol-3-yl)indolin-2-one (3c). Interestingly excellent yields were obtained for products synthesized from the reactions between N-allyl, N-benzyl, N-propargyl and $\mathrm{N}$-methyl isatins and 5-methoxy indole which is electron rich at the third position. Thus, N-allyl-3-hydroxy-3-(5-methoxy-indol-3-yl)indolin-2-one (8c), N-benzyl-3-hydroxy-3-(5-methoxy-indol-3-yl) indolin-2-one (12c), N-propargyl-3-hydroxy-3-(5-methoxy-indol-3-yl)indolin-2-one (14c) and N-methyl-3-hydroxy3-(5-methoxy-indol-3-yl)indolin-2-one (15c) gave yields of 97\%, 98\%, 89\% and 96\% respectively. 76\% yield was obtained in the reaction of 2-methyl indole with simple isatin to give 3-hydroxy-3-(2-methyl-1H-indole3-yl)indolin-2-one (2c), while $\mathrm{N}$-allyl isatin and $\mathrm{N}$-benzyl isatin gave $\mathrm{N}$-allyl-3-hydroxy-3-(2-methyl-indol-3yl)indolin-2-one (7c) and N-benzyl-3-hydroxy-3-(2-methyl-indol-3-yl)indolin-2-one (11c) with $90 \%$ and $92 \%$ yields respectively. Moderate yields were obtained in the case of $\mathrm{N}$-methyl indole with various isatins. The yields were 56\% for N-allyl-3-hydroxy-3-(1-methyl-indol-3-yl)indolin-2-one (6c) 56\% for N-benzyl-3-hydroxy3-(1-methyl-indol-3-yl)indolin-2-one (10c) and 58\% for N-propargyl-3-hydroxy-3-(1-methyl-indol-3-yl) indolin-2-one (13c). No product was obtained in the case of electron withdrawing nitro group present at fifth position of the indole ring with simple isatin even after a prolonged reaction time of 10 minutes and at a temperature of $100^{\circ} \mathrm{C}$ (Table 1).

The proposed reaction mechanism for the synthesis of 3-hydroxy-3-(1H-indol-3-yl)indolin-2-one (1c) is shown in Scheme 2.

Entry 8 (Table 1) was selected as a representative example for comparing reaction rates in various solvents such as acetonitrile (ACN), 1,4-dioxane, water $\left(\mathrm{H}_{2} \mathrm{O}\right)$, ethanol (EtOH) and 1,2-dichloroethane (1,2-DCE). Moderate yield was observed only in the case of ACN (53\%) in five minutes which improved to $96 \%$ in a reaction time of 15 minutes. Comparatively less yields were observed in the case of $\mathrm{H}_{2} \mathrm{O}$ (46\%), EtOH (46\%), 1,2-DCE (36\%) and 1,4-dioxane (15\%).

\section{Synthesis of Unsymmetrical 3,3-Di(indolyl)indolin-2-ones}

Formation of symmetrical diindolyl was observed as a major product at $100^{\circ} \mathrm{C}$ in the synthesis of 3-hydroxy-3(1H-indol-3-yl)indolin-2-one (1c). The same conditions were adopted for synthesizing unsymmetrical 3,3-di (indolyl)indolin-2-ones. For optimization, 5-methoxy-3-hydroxy-3-(1H-indol-3-yl)indolin-2-one (1d) [1 equivalent] and indole [1.2 equivalents] were selected. At a microwave oven temperature of $150^{\circ} \mathrm{C}$ and irradiation for 5 minutes, 53\% product 3-(5-methoxy-1H-indol-3-yl)-3-(1H-indol-3-yl)indolin-2-one (1e) was formed. At a temperature higher than $150^{\circ} \mathrm{C}$ and a reaction time of 10 minutes, no significant increase in the yield was observed. When the same conditions were applied to the reaction [i.e. $150^{\circ} \mathrm{C}$ for 10 minutes] using various $\mathrm{N}$ substituted isatins, good yields were obtained in all the cases (Table 2, Scheme 3).

All the compounds were characterized by IR, ${ }^{1} \mathrm{H},{ }^{13} \mathrm{C}$ NMR and HRMS. To the best of our knowledge com- 

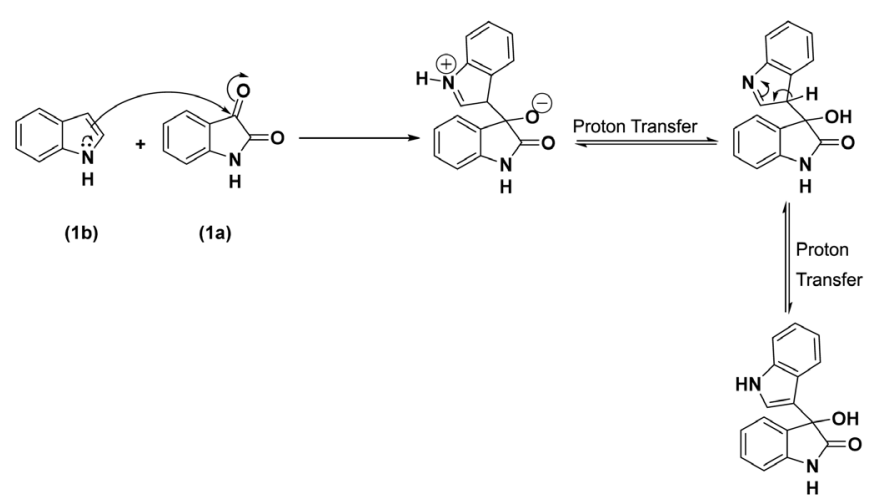

(1c)

Scheme 2. Proposed mechanism for the formation of 3-indolyl-3-hydroxy oxindole.

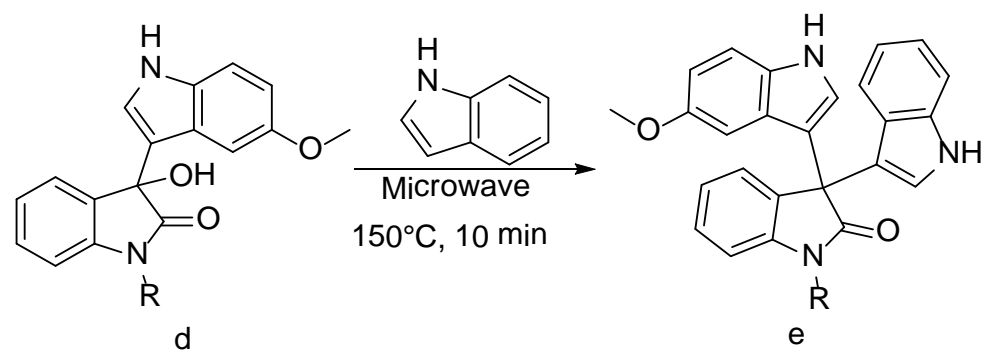

Scheme 3. Synthesis of various unsymmetrical 3,3-di(indolyl)indolin-2-ones.

Table 1. Synthesis of various 3-indolyl-3-hydroxy oxindoles.

\begin{tabular}{|c|c|c|c|c|}
\hline SI. No. & Isatin a & Indole $\mathbf{b}$ & Product $\mathbf{c}$ & Yield $\%^{[\mathrm{a}]}$ \\
\hline 1 & $\mathrm{R}=\mathrm{H}$ & $\mathrm{R}_{1}=\mathrm{H}, \mathrm{R}_{2}=\mathrm{H}, \mathrm{R}_{3}=\mathrm{H}$ & $1 \mathrm{c}$ & 31 \\
\hline 2 & $\mathrm{R}=\mathrm{H}$ & $\mathrm{R}_{1}=\mathrm{H}, \mathrm{R}_{2}=\mathrm{Me}, \mathrm{R}_{3}=\mathrm{H}$ & 2c & 76 \\
\hline 3 & $\mathrm{R}=\mathrm{H}$ & $\mathrm{R}_{1}=\mathrm{H}, \mathrm{R}_{2}=\mathrm{H}, \mathrm{R}_{3}=\mathrm{OMe}$ & 3с & 64 \\
\hline 4 & $\mathrm{R}=\mathrm{H}$ & $\mathrm{R}_{1}=\mathrm{H}, \mathrm{R}_{2}=\mathrm{H}, \mathrm{R}_{3}=\mathrm{NO}_{2}$ & $4 c$ & 00 \\
\hline 5 & $\mathrm{R}=$ allyl & $\mathrm{R}_{1}=\mathrm{H}, \mathrm{R}_{2}=\mathrm{H}, \mathrm{R}_{3}=\mathrm{H}$ & $5 c$ & 34 \\
\hline 6 & $\mathrm{R}=$ allyl & $\mathrm{R}_{1}=\mathrm{Me}, \mathrm{R}_{2}=\mathrm{H}, \mathrm{R}_{3}=\mathrm{H}$ & $6 c$ & 56 \\
\hline 7 & $\mathrm{R}=$ allyl & $\mathrm{R}_{1}=\mathrm{H}, \mathrm{R}_{2}=\mathrm{Me}, \mathrm{R}_{3}=\mathrm{H}$ & 7c & 90 \\
\hline 8 & $\mathrm{R}=$ allyl & $\mathrm{R}_{1}=\mathrm{H}, \mathrm{R}_{2}=\mathrm{H}, \mathrm{R}_{3}=\mathrm{OMe}$ & $8 c$ & 97 \\
\hline 9 & $\mathrm{R}=$ benzyl & $\mathrm{R}_{1}=\mathrm{H}, \mathrm{R}_{2}=\mathrm{H}, \mathrm{R}_{3}=\mathrm{H}$ & 9c & 40 \\
\hline 10 & $\mathrm{R}=$ benzyl & $\mathrm{R}_{1}=\mathrm{H}, \mathrm{R}_{2}=\mathrm{H}, \mathrm{R}_{3}=\mathrm{H}$ & 10c & 56 \\
\hline 11 & $\mathrm{R}=$ benzyl & $\mathrm{R}_{1}=\mathrm{H}, \mathrm{R}_{2}=\mathrm{Me}, \mathrm{R}_{3}=\mathrm{H}$ & $11 \mathrm{c}$ & 92 \\
\hline 12 & $\mathrm{R}=$ benzyl & $\mathrm{R}_{1}=\mathrm{H}, \mathrm{R}_{2}=\mathrm{H}, \mathrm{R}_{3}=\mathrm{OMe}$ & $12 \mathrm{c}$ & 98 \\
\hline 13 & $\mathrm{R}$ = propargyl & $\mathrm{R}_{1}=\mathrm{Me}, \mathrm{R}_{2}=\mathrm{H}, \mathrm{R}_{3}=\mathrm{H}$ & $13 c$ & 58 \\
\hline 14 & $\mathrm{R}=$ propargyl & $\mathrm{R}_{1}=\mathrm{H}, \mathrm{R}_{2}=\mathrm{H}, \mathrm{R}_{3}=\mathrm{OMe}$ & $14 \mathrm{c}$ & 89 \\
\hline 15 & $\mathrm{R}$ = propargyl & $\mathrm{R}_{1}=\mathrm{H}, \mathrm{R}_{2}=\mathrm{H}, \mathrm{R}_{3}=\mathrm{OMe}$ & $15 c$ & 96 \\
\hline
\end{tabular}

[a] Isolated yield. 
Table 2. Synthesis of various unsymmetrical 3,3-di(indolyl)indolin-2-ones.

\begin{tabular}{cccc}
\hline SI.No. & Substrate $(\mathbf{R}) \mathbf{d}$ & Product e & Yield \% $^{\text {[a] }}$ \\
\hline 1 & $\mathrm{H}$ & $1 \mathrm{e}$ & 53 \\
2 & methyl & $2 \mathrm{e}$ & 72 \\
3 & allyl & $3 \mathrm{e}$ & 78 \\
4 & benzyl & $4 \mathrm{e}$ & 77 \\
5 & propargyl & $5 \mathrm{e}$ & 77 \\
\hline
\end{tabular}

[a] Isolated yield.

pounds N-Allyl-3-hydroxy-3-(1-methyl-indol-3-yl)indolin-2-one (6c), N-Allyl-3-hydroxy-3-(5-methoxy-indol3-yl)indolin-2-one (8c), N-Benzyl-3-hydroxy-3-(1-methyl-indol-3-yl)indolin-2-one (10c), N-Propargyl-3-hydroxy-3-(1-methyl-indol-3-yl)indolin-2-one (13c), N-propargyl-3-hydroxy-3-(5-methoxy-indol-3-yl)indolin-2one (14c); 3-(5-methoxy-1H-indol-3-yl)-3-(1H-indol-3-yl)indolin-2-one(1e), 3-1-methyl(5-methoxy-1H-in-dol-3yl)-3-(1H-indol-3-yl)indolin-2-one (2e), 3-1-allyl(5-methoxy-1H-indol-3-yl)-3-(1H-indol-3-yl)indolin-2-one (3e), 3-1-benzyl(5-methoxy-1H-indol-3-yl)-3-(1H-indol-3-yl)indolin-2-one (4e) and 3-1-(prop-2-ynyl)(5-methoxy- $1 H$ indol-3-yl)-3-(1H-indol-3-yl)indolin-2-one (5e) are reported here for the first time.

\section{Material and Methods}

Chemicals were obtained from Spectrochem, and used without further purification. All known products were identified by comparison of their physical and spectral data with those of authentic samples. ${ }^{1} \mathrm{H}$ and ${ }^{13} \mathrm{C}$ NMR spectra were recorded on a Bruker AV-400 spectrometer operating at 400 and $100 \mathrm{MHz}$, respectively. The spectra were calibrated on the solvent residual peak (DMSO-d6: $\delta=2.50 \mathrm{ppm}$ for ${ }^{1} \mathrm{H}$ and $\delta=39.52 \mathrm{ppm}$ for ${ }^{13} \mathrm{C}$; $\mathrm{CDCl}_{3}: \delta=7.26 \mathrm{ppm}$ for ${ }^{1} \mathrm{H}$ and $\delta=77.0 \mathrm{ppm}$ for ${ }^{13} \mathrm{C}$ ). Analytical thin layer chromatography (TLC) was performed on Kieselgel 60 F254 aluminum sheets (Merck 1.05554). Column chromatography was performed on silica gel (100 - 200 mesh). FT-IR spectra were recorded on a Nicolet-6700. High-resolution mass spectra were recorded with Thermo Scientific-Orbitrap Elite (Electro spray Ionization). Anton Paar microwave synthesizer was used at $600 \mathrm{rpm}$ and temperature was kept constant at $70^{\circ} \mathrm{C}$ and $150^{\circ} \mathrm{C}$.

\subsection{General Procedure for the Synthesis of 3-Indolyl-3-hydroxy Oxindoles (1c to 15c)}

A mixture of the isatin $(0.6797 \mathrm{mmol}, 100 \mathrm{mg})$ and indole $(0.8156 \mathrm{mmol}, 96 \mathrm{mg})$ was added to a microwave-oven reaction vial then irradiated for 5 minutes at $70^{\circ} \mathrm{C}$. After completion of the reaction (as indicated by TLC) the residue was washed with $2 \mathrm{~mL}$ of DCM and mixed with silica gel and then evaporated the solvent under reduced pressure, and the mixture was purified by column chromatography using EtOAc in hexanes. All the known products have spectral and physical data consistent with those reported in literatures.

\subsubsection{3-Hydroxy-3-(1H-indol-3-yl)indolin-2-one (1c)}

White solid; mp: 294 - 296 (lit ${ }^{26}: 294^{\circ} \mathrm{C}-296^{\circ} \mathrm{C}$ ). ${ }^{1} \mathrm{H}$ NMR (400 MHz, DMSO-d ${ }_{6}$ ): $\delta 10.98$ (s, $\left.1 \mathrm{H}\right), 10.34$ (s, 1H), 7.35 (t, $J=9.6 \mathrm{~Hz}, 2 \mathrm{H}), 7.27-7.24$ (m, 2H), 7.09 (d, $J=2.4 \mathrm{~Hz}, 1 \mathrm{H}), 7.03-7.02$ (m, 1H), 6.98 - 6.86 (m, 4H), 6.36 (s, 1H). ${ }^{13} \mathrm{C}$ NMR (100 MHz, DMSO-d $\left.{ }_{6}\right): \delta 178.5,141.7,136.8,133.5,129.1,124.9,124.8,123.5$, 121.7, 121.1, 120.3, 118.5, 115.5, 111.5, 109.6, 74.9. IR (KBr): v 3264, 1711, 1617, 1549, 1467, 1424, 1335, 1226, 1184, 1107, 1071, 938, 910, 745, 686, $655 \mathrm{~cm}^{-1}$. HRMS: Calcd for $\mathrm{C}_{16} \mathrm{H}_{12} \mathrm{O}_{2} \mathrm{~N}_{2}[\mathrm{M}+\mathrm{Na}]^{+} 287.0899$, found 287.0795 .

\subsubsection{3-Hydroxy-3-(2-methyl-1H-indol-3-yl)indolin-2-one (2c)}

White solid; mp: $178^{\circ} \mathrm{C}-180^{\circ} \mathrm{C}\left(\mathrm{lit}^{26}: 176^{\circ} \mathrm{C}-178^{\circ} \mathrm{C}\right) .{ }^{1} \mathrm{H}$ NMR (400 MHz, DMSO- $\left.d_{6}\right): \delta 10.86(\mathrm{~s}, 1 \mathrm{H}), 10.33(\mathrm{~s}$, $1 \mathrm{H}), 7.25-7.17(\mathrm{~m}, 3 \mathrm{H}), 6.96-6.88(\mathrm{~m}, 4 \mathrm{H}), 6.74-6.70(\mathrm{~m}, 1 \mathrm{H}), 6.26(\mathrm{~s}, 1 \mathrm{H}), 2.34(\mathrm{~s}, 3 \mathrm{H}) .{ }^{13} \mathrm{C}$ NMR $(100$ MHz, DMSO-d ${ }_{6}$ ): $\delta 178.7,141.6,134.8,134.1,133.4,129.0,126.6,124.9,121.7,119.8,119.2,118.2,110.2$, 109.6, 109.4, 75.8, 13.3. IR (KBr): v 3348, 3229, 3034, 1693, 1611, 1487, 1454, 1431, 1374, 1345, 1291, 1239 , 1211, 1163, 1024, 996, 954, 927, 893,853, 832, 737, $696 \mathrm{~cm}^{-1}$. HRMS: Calcd for $\mathrm{C}_{17} \mathrm{H}_{14} \mathrm{O}_{2} \mathrm{~N}_{2}[\mathrm{M}+\mathrm{Na}]^{+}$ 301.0948; found 301.0953. 
3.1.3. 5-Methoxy-3-hydroxy-3-(1H-Indol-3-yl)indolin-2-one (3c)

White solid; mp: $196^{\circ} \mathrm{C}-198^{\circ} \mathrm{C}\left(\mathrm{lit}^{26}: 196^{\circ} \mathrm{C}-198^{\circ} \mathrm{C}\right) .{ }^{1} \mathrm{H}$ NMR (400 MHz, DMSO- $\left.d_{6}\right): \delta 10.84(\mathrm{~s}, 1 \mathrm{H}), 10.33$ (s, 1H), $7.28-7.23(\mathrm{~m}, 3 \mathrm{H}), 7.02$ (d, $J=2.8 \mathrm{~Hz}, 1 \mathrm{H}), 6.98(\mathrm{t}, J=7.6 \mathrm{~Hz}, 1 \mathrm{H}), 6.92(\mathrm{~d}, J=7.26 \mathrm{~Hz}, 1 \mathrm{H}), 6.86(\mathrm{~d}, J$ $=2 \mathrm{~Hz}, 6.71(\mathrm{dd}, J=2.4 \mathrm{~Hz}, J=8.8 \mathrm{~Hz}, 1 \mathrm{H}), 6.34(\mathrm{~s}, 1 \mathrm{H}), 5.76(\mathrm{~s}, 1 \mathrm{H}), 3.62(\mathrm{~s}, 3 \mathrm{H}) .{ }^{13} \mathrm{C}$ NMR $(100 \mathrm{MHz}$, DMSO-d $\left.\mathrm{d}_{6}\right): \delta 178.5,152.7,141.7,133.4,132.0,129.0,125.4,124.8,124.2,121.7,115.0,112.0,110.9,109.6$, 102.7, 75.0, 55.2. IR (KBr): v 3318, 3166, 1701, 1614, 1581, 1465, 1433, 1344, 1304, 1235, 1207, 1173, 1039, 927, 837, 779, 752, $650 \mathrm{~cm}^{-1}$. HRMS: Calcd for $\mathrm{C}_{17} \mathrm{H}_{14} \mathrm{O}_{3} \mathrm{~N}_{2}$ [M + Na] $]^{+}$317.0897; found 317.0902.

3.1.4. N-allyl-3-hydroxy-3-(1H-indol-3-yl)indolin-2-one (5c)

White solid; mp: $148^{\circ} \mathrm{C}-150^{\circ} \mathrm{C} .{ }^{1} \mathrm{H}$ NMR $\left(400 \mathrm{MHz}\right.$, DMSO- $\left.d_{6}\right): \delta 11.01(\mathrm{~s}, 1 \mathrm{H}), 7.35-7.33(\mathrm{~m}, 4 \mathrm{H}), 7.06$ $7.04(\mathrm{~m}, 4 \mathrm{H}), 6.89-6.86(\mathrm{~m}, 1 \mathrm{H}), 6.49(\mathrm{~s}, 1 \mathrm{H}), 5.89-5.82(\mathrm{~m}, 1 \mathrm{H}), 5.24-5.16(\mathrm{~m}, 2 \mathrm{H}), 4.38-4.27(\mathrm{~m}, 2 \mathrm{H}) .{ }^{13} \mathrm{C}$ NMR (100 MHz, DMSO-d $\left.\mathrm{d}_{6}\right): \delta 176.4,142.2,136.8,132.7,132.0,129.0,124.9,124.5,123.6,122.3,121.1$, 120.4, 118.5, 117.0, 115.2, 111.5, 109.1, 74.6, 41.4. IR (KBr): v 3219, 3056, 1688, 1603, 1485, 1459, 1435, 1365, 1332, 1294, 1241, 1223, 1182, 1104, 981, 921, 905, 826, 735, $682 \mathrm{~cm}^{-1}$. HRMS: Calcd for $\mathrm{C}_{19} \mathrm{H}_{16} \mathrm{O}_{2} \mathrm{~N}_{2}$ $[\mathrm{M}+\mathrm{Na}]^{+}$327.1109; found 327.1110.

\subsubsection{N-allyl-3-hydroxy-3-(1-methyl-indol-3-yl)indolin-2-one (6c)}

White solid; mp: $136^{\circ} \mathrm{C}-138^{\circ} \mathrm{C} .{ }^{1} \mathrm{H}$ NMR $\left(400 \mathrm{MHz}, \mathrm{CDCl}_{3}\right): \delta 7.67(\mathrm{~d}, J=8 \mathrm{~Hz}, 1 \mathrm{H}), 7.52(\mathrm{~d}, J=7.2 \mathrm{~Hz}, 1 \mathrm{H})$, 7.33 - 7.19 (m, 3H), 7.09 - 7.05 (m, 2H), 6.97 (s, 1H), 6.90 (d, J = $7.6 \mathrm{~Hz}, 1 \mathrm{H}), 5.91$ - 5.81 (m, 1H), 5.30 - 5.22 (m, 2H), 4.44 (dd, $J=4.8 \mathrm{~Hz}, J=16 \mathrm{~Hz}, 1 \mathrm{H}), 4.28(\mathrm{dd}, J=4.8 \mathrm{~Hz}, 16.4 \mathrm{~Hz}, 1 \mathrm{H}), 3.69(\mathrm{~s}, 3 \mathrm{H}), 3.42(\mathrm{~m}, 1 \mathrm{H}) .{ }^{13} \mathrm{C}$ NMR (100 MHz, $\left.\mathrm{CDCl}_{3}\right): \delta$ 176.9, 142.4, 137.7, 131.2, 129.6, 127.7, 125.4, 124.9, 123.1, 122.1, 120.8, 119.7, 117.8, 113.8, 109.5, 109.4, 75.5, 42.5, 32.8. IR (KBr): v 3349, 3046, 1699, 1606, 1462, 1412, 1365, 1329, 1208, 1178, 1137, 1113, 1072, 984, 934, 888, 743, $669 \mathrm{~cm}^{-1}$. HRMS: Calcd for $\mathrm{C}_{20} \mathrm{H}_{18} \mathrm{O}_{2} \mathrm{~N}_{2}[\mathrm{M}+\mathrm{Na}]^{+} 341.1260$; found 341.1261.

\subsubsection{N-allyl-3-hydroxy-3-(2-methyl-indol-3-yl)indolin-2-one (7c)}

White solid; mp: $164^{\circ} \mathrm{C}-166^{\circ} \mathrm{C}\left(\mathrm{lit}^{26}: 164^{\circ} \mathrm{C}-166^{\circ} \mathrm{C}\right) .{ }^{1} \mathrm{H}$ NMR $\left(400 \mathrm{MHz}\right.$, DMSO-d $\left.\mathrm{d}_{6}\right): \delta 10.91(\mathrm{~s}, 1 \mathrm{H}), 7.32(\mathrm{t}$, $J=7.6 \mathrm{~Hz}, 1 \mathrm{H}), 7.26(\mathrm{~d}, J=7.2 \mathrm{~Hz}, 1 \mathrm{H}), 7.19(\mathrm{~d}, J=8 \mathrm{~Hz}, 1 \mathrm{H}), 7.01(\mathrm{t}, J=7.6 \mathrm{~Hz}, 2 \mathrm{H}), 6.92-6.85(\mathrm{~m}, 2 \mathrm{H})$, $6.71(\mathrm{t}, J=7.2 \mathrm{~Hz}, 1 \mathrm{H}), 6.40(\mathrm{~s}, 1 \mathrm{H}), 5.91-5.81(\mathrm{~m}, 1 \mathrm{H}), 5.27$ - $5.16(\mathrm{~m}, 2 \mathrm{H}), 4.39$ - $4.26(\mathrm{~m}, 2 \mathrm{H}), 2.38(\mathrm{~s}, 3 \mathrm{H})$. ${ }^{13} \mathrm{C}$ NMR (100 MHz, DMSO-d $\left.\mathrm{d}_{6}\right): \delta 176.6,142.2,134.8,133.7,133.3,131.9,129.0,126.6,124.7,122.4,119.8$, 119.2, 118.2, 117.1, 110.3, 109.1, 75.5, 41.5, 13.3. IR (KBr): v 3401, 1703, 1607, 1523, 1488, 1461, 1431, 1370, 1331, 1304, 1222, 1181, 982, 931, 895, 759, $673 \mathrm{~cm}^{-1}$. HRMS: Calcd for $\mathrm{C}_{20} \mathrm{H}_{18} \mathrm{O}_{2} \mathrm{~N}_{2}[\mathrm{M}+\mathrm{Na}]^{+} 341.1261$; found 341.1266 .

\subsubsection{N-allyl-3-hydroxy-3-(5-methoxy-indol-3-yl)indolin-2-one (8c)}

White solid; mp: $102^{\circ} \mathrm{C}-104^{\circ} \mathrm{C} .{ }^{1} \mathrm{H}$ NMR (400 MHz, DMSO- $\left.d_{6}\right): \delta 10.88$ (s, $\left.1 \mathrm{H}\right), 7.34$ (brs, 2H), 7.24 (d, $J=$ $8.8 \mathrm{~Hz}, 1 \mathrm{H}), 7.08-7.06(\mathrm{~m}, 3 \mathrm{H}), 6.78(\mathrm{~s}, 1 \mathrm{H}), 6.70(\mathrm{~d}, J=8.4 \mathrm{~Hz}, 1 \mathrm{H}), 6.48(\mathrm{~s}, 1 \mathrm{H}), 5.89-5.82(\mathrm{~m}, 1 \mathrm{H}), 5.25(\mathrm{~s}$, $1 \mathrm{H}), 5.18(\mathrm{t}, J=10.8 \mathrm{~Hz}, 1 \mathrm{H}), 4.32(\mathrm{dd}, J=14 \mathrm{~Hz}, J=35.6 \mathrm{~Hz}, 2 \mathrm{H}), 3.60(\mathrm{~s}, 3 \mathrm{H}) .{ }^{13} \mathrm{C}$ NMR $(100 \mathrm{MHz}$, DMSO-d $\left.{ }_{6}\right): \delta 176.4,152.8,142.2,132.6,132.0,131.9,129.1,125.3,124.6,124.3,122.4,117.0,114.7,112.1$, 111.2, 109.0, 102.2, 74.6, 55.1, 41.5. IR (KBr): v 3324, 1702, 1611, 1485, 1463, 1435, 1361, 1210, 1177, 1109, 1064, 990, 9224, 899, 840, 805, 752, 704, 674, $631 \mathrm{~cm}^{-1}$. HRMS: Calcd for $\mathrm{C}_{20} \mathrm{H}_{18} \mathrm{O}_{3} \mathrm{~N}_{2}[\mathrm{M}+\mathrm{Na}]^{+} 357.1215$; found 357.1202.

\subsubsection{N-benzyl-3-hydroxy-3-(1H-indol-3-yl)indolin-2-one (9c)}

White solid; mp: $120^{\circ} \mathrm{C}-124^{\circ} \mathrm{C}\left(\mathrm{lit}^{26}: 120^{\circ} \mathrm{C}-124^{\circ} \mathrm{C}\right) .{ }^{1} \mathrm{H}$ NMR $\left(400 \mathrm{MHz}\right.$, DMSO- $\left.d_{6}\right): \delta 11.03(\mathrm{~s}, 1 \mathrm{H}), 7.37-$ 7.24 (m, 9H), 7.10 (s, 1H), $7.05-6.96$ (m, 3H), 6.82 (t, $J=7.6 \mathrm{~Hz}, 1 \mathrm{H}), 6.57$ (s, 1H), $4.92(\mathrm{~s}, 2 \mathrm{H}) .{ }^{13} \mathrm{C}$ NMR (100 MHz, DMSO-d ${ }_{6}$ ): $\delta$ 176.8, 142.1, 136.8, 136.4, 132.7, 129.0, 128.5, 127.4, 124.8, 124.5, 124.5, 123.6, 122.5, 121.1, 120.4, 118.4, 115.1, 111.5, 109.1, 74.7, 42.7. IR (KBr): v 3296, 3029, 1697, 1607, 1488, 1458, 1428, 1342, 1238, 1212, 1166, 1067, 991, 901, 738, $694 \mathrm{~cm}^{-1}$. HRMS: Calcd for $\mathrm{C}_{23} \mathrm{H}_{18} \mathrm{O}_{2} \mathrm{~N}_{2}[\mathrm{M}+\mathrm{Na}]^{+}$ 377.1266; found 377.1251.

3.1.9. N-benzyl-3-hydroxy-3-(1-methyl-indol-3-yl)indolin-2-one (10c)

White solid; mp: $126^{\circ} \mathrm{C}-128^{\circ} \mathrm{C} .{ }^{1} \mathrm{H}$ NMR (400 MHz, DMSO- $\left.d_{6}\right): \delta 7.38-7.25$ (m, 9H), $7.12-7.10$ (m, 2H), 
7.02 (t, $J=7.6 \mathrm{~Hz}, 1 \mathrm{H}), 6.97$ (d, $J=8 \mathrm{~Hz}, 1 \mathrm{H}), 6.87$ (t, $J=7.6 \mathrm{~Hz}, 1 \mathrm{H}), 6.54$ (s, 1H), 4.91 (s, 2H), 3.73 (s, 3H).

${ }^{13}$ C NMR (100 MHz, DMSO-d $\left.\mathrm{d}_{6}\right): \delta 176.6,142.1,137.2,136.3,132.6,129.0,128.4,127.9,127.3,125.1,124.5$, 122.4, 121.1, 120.6, 118.5, 114.3, 109.6, 109.1, 74.5, 42.7, 32.3. IR (KBr): v 3302, 1693, 1610, 1549, 1486, 1464, 1373, 1342, 1211, 1164, 1112, 1077, 990, 928, 893, 855, 747, 702, 669, $628 \mathrm{~cm}^{-1}$. HRMS: Calcd for $\mathrm{C}_{24} \mathrm{H}_{20} \mathrm{O}_{2} \mathrm{~N}_{2}[\mathrm{M}+\mathrm{Na}]^{+}$391.1422; found 391.1420.

\subsubsection{N-benzyl-3-hydroxy-3-(2-methyl-indol-3-yl)indolin-2-one (11c)}

White solid; mp: $96^{\circ} \mathrm{C}-98^{\circ} \mathrm{C} .{ }^{1} \mathrm{H}$ NMR (400 MHz, DMSO- $\left.d_{6}\right): \delta 10.91(\mathrm{~s}, 1 \mathrm{H}), 7.36$ - $7.18(\mathrm{~m}, 7 \mathrm{H}), 7.19(\mathrm{~d}, J=$ $8 \mathrm{~Hz}, 1 \mathrm{H}), 6.99$ (t, $J=7.2 \mathrm{~Hz}, 2 \mathrm{H}), 6.92-6.88(\mathrm{~m}, 1 \mathrm{H}), 6.80$ (d, $J=8 \mathrm{~Hz}, 1 \mathrm{H}), 6.68$ - $6.64(\mathrm{~m}, 1 \mathrm{H}), 6.47$ (s, 1H), $4.92(\mathrm{ABq}, J=16 \mathrm{~Hz}, J=20 \mathrm{~Hz}, 2 \mathrm{H}), 2.37$ (s, 3H). ${ }^{13} \mathrm{C}$ NMR $\left(100 \mathrm{MHz}\right.$, DMSO-d $\left.{ }_{6}\right): \delta 177.0,142.1,136.3$, 134.8, 133.7, 133.4, 129.0, 128.5, 127.5, 126.5, 124.8, 122.5, 119.8, 119.2, 118.1, 110.3, 109.1, 109.2, 109.1, 75.6, 42.7, 13.3. IR (KBr): v 3314, 3288, 3092, 1700, 1610, 1551, 1530, 1486, 1461, 1430, 1350, 1302, 1237, 1173, 1074, 993, 917, 745, 698, $634 \mathrm{~cm}^{-1}$. HRMS: Calcd for $\mathrm{C}_{24} \mathrm{H}_{20} \mathrm{O}_{2} \mathrm{~N}_{2}[\mathrm{M}+\mathrm{Na}]^{+}$391.1417; found 391.1418.

\subsubsection{N-benzyl-3-hydroxy-3-(5-methoxy-indol-3-yl)indolin-2-one (12c)}

White solid; mp: $204^{\circ} \mathrm{C}-206^{\circ} \mathrm{C} .{ }^{1} \mathrm{H}$ NMR (400 MHz, DMSO- $\left.d_{6}\right): \delta 10.89(\mathrm{~s}, 1 \mathrm{H}), 7.30-7.23(\mathrm{~m}, 8 \mathrm{H}), 7.05-$ $7.02(\mathrm{~m}, 2 \mathrm{H}), 6.97(\mathrm{~d}, J=7.6 \mathrm{~Hz}, 1 \mathrm{H}), 6.70-6.67(\mathrm{~m}, 2 \mathrm{H}), 6.55(\mathrm{~s}, 1 \mathrm{H}), 4.91$ (dd, $J=15.6 \mathrm{~Hz}, J=22 \mathrm{~Hz}, 2 \mathrm{H})$, 3.49 (s, $3 \mathrm{H}) .{ }^{13} \mathrm{C}$ NMR $\left(100 \mathrm{MHz}\right.$, DMSO-d $\left.\mathrm{d}_{6}\right): \delta 176.8,152.7,142.2,136.4,132.7,131.9,129.1,128.5,127.3$, 125.2, 124.7, 124.3, 122.5, 114.7, 112.1, 111.2, 109.1, 102.2, 74.7, 54.9, 42.6. IR (KBr): v 3354, 3283, 3047, 1702, 1611, 1582, 1527, 1486, 1461, 1350, 1306, 1211, 1174, 1065, 995, 931, 902, 854, 751, 698, $673 \mathrm{~cm}^{-1}$. HRMS: Calcd for $\mathrm{C}_{24} \mathrm{H}_{20} \mathrm{O}_{3} \mathrm{~N}_{2}[\mathrm{M}+\mathrm{Na}]^{+}$407.1372; found 407.1357.

\subsubsection{N-propargyl-3-hydroxy-3-(1-methyl-indol-3-yl)indolin-2-one (13c)}

White solid; mp: $158^{\circ} \mathrm{C}-162^{\circ} \mathrm{C} .{ }^{1} \mathrm{H}$ NMR $\left(400 \mathrm{MHz}\right.$, DMSO- $\left.d_{6}\right): \delta 7.41-7.33(\mathrm{~m}, 4 \mathrm{H}), 7.19(\mathrm{~d}, J=8 \mathrm{~Hz}, 1 \mathrm{H})$, $7.13-7.08(\mathrm{~m}, 3 \mathrm{H}), 6.91(\mathrm{t}, J=7.2 \mathrm{~Hz}, 1 \mathrm{H}), 6.60(\mathrm{~s}, 1 \mathrm{H}), 4.56(\mathrm{ABq}, J=16.4 \mathrm{~Hz}, J=33.2 \mathrm{~Hz}, 2 \mathrm{H}), 3.72(\mathrm{~s}, 3$ $\mathrm{H}), 3.31(\mathrm{~s}, 1 \mathrm{H}) .{ }^{13} \mathrm{C}$ NMR (100 MHz, DMSO-d $\left.\mathrm{d}_{6}\right): \delta 175.7,141.2,137.2,132.5,129.2,127.9,125.1,124.6$, $122.8,121.3,120.6,118.7,114.2,109.7,109.3,78.0,74.5,32.4,28.8$. IR (KBr): v 3322, 3254, 1711, 1609, 1535, 1463, 1426, 1369, 1335, 1244, 1213, 1170, 1110, 1071, 997, 934,892, 744, 671, 628. HRMS: Calcd for $\mathrm{C}_{20} \mathrm{H}_{16} \mathrm{O}_{2} \mathrm{~N}_{2}[\mathrm{M}+\mathrm{Na}]^{+}$339.1109; found 339.1097.

\subsubsection{N-propargyl-3-hydroxy-3-(5-methoxy-indol-3-yl)indolin-2-one (14c)}

White solid; mp: $181^{\circ} \mathrm{C}-184^{\circ} \mathrm{C} .{ }^{1} \mathrm{H}$ NMR (400 MHz, DMSO- $\left.d_{6}\right): \delta 10.9(\mathrm{~s}, 1 \mathrm{H}), 7.41(\mathrm{t}, J=7.6 \mathrm{~Hz}, 1 \mathrm{H}), 7.33$ (d, $J=7.6 \mathrm{~Hz}, 1 \mathrm{H}), 7.25-7.18(\mathrm{~m}, 2 \mathrm{H}), 7.12-7.07(\mathrm{~m}, 2 \mathrm{H}), 6.75(\mathrm{~s}, 1 \mathrm{H}), 6.70(\mathrm{~d}, J=8.4 \mathrm{~Hz}, 1 \mathrm{H}), 6.55(\mathrm{~s}, 1 \mathrm{H})$, 4.57 (ABq, $J=18 \mathrm{~Hz}, J=45.2 \mathrm{~Hz}, 2 \mathrm{H}) .{ }^{13} \mathrm{C}$ NMR (100 MHz, DMSO-d $\left.{ }_{6}\right): \delta 175.8,152.9,141.3,132.5,131.9$, $129.1,125.1,124.6,124.3,122.8,114.5,112.1,111.3,109.2,102.0,78.1,74.7,74.5,55.2$ 28.8. IR (KBr): v 3411, 3325, 3276, 3089, 3052, 1705, 1611, 1583, 1530, 1486, 1463, 1438, 1360, 1291, 1242, 1209, 1177, 1111, 1059, 991, 924, 894, 835, 752, 702, $667 \mathrm{~cm}^{-1}$. HRMS: Calc. for $\mathrm{C}_{20} \mathrm{H}_{16} \mathrm{O}_{3} \mathrm{~N}_{2}[\mathrm{M}+\mathrm{Na}]^{+}$Calcd 355.1059; found 355.1047.

\subsubsection{N-methyl-3-hydroxy-3-(5-methoxy-indol-3-yl)indolin-2-one (15c)}

White solid; mp: $102^{\circ} \mathrm{C}-104^{\circ} \mathrm{C} .{ }^{1} \mathrm{H}$ NMR (400 MHz, DMSO- $\left.d_{6}\right): \delta 10.85(\mathrm{~s}, 1 \mathrm{H}), 7.39-7.31(\mathrm{~m}, 2 \mathrm{H}), 7.23$ (d, $J$ $=8.8 \mathrm{~Hz}, 1 \mathrm{H}), 7.09-7.06(\mathrm{~m}, 2 \mathrm{H}), 7.01(\mathrm{~s}, 1 \mathrm{H}), 6.81(\mathrm{~s}, 1 \mathrm{H}), 6.70(\mathrm{~d}, J=8.8 \mathrm{~Hz}, 1 \mathrm{H}), 6.40(\mathrm{~s}, 1 \mathrm{H}), 3.62(\mathrm{~s}, 3 \mathrm{H})$, $3.16(\mathrm{~s}, 3 \mathrm{H}) .{ }^{13} \mathrm{C}$ NMR $\left(100 \mathrm{MHz}, \mathrm{DMSO}_{\mathrm{d}}\right): \delta 176.6,152.7,143.1,132.7,131.9,129.2,125.3,124.4,124.2$, 122.4, 114.7, 112.1, 111.0, 108.4, 102.3, 74.7, 55.1, 25.9. IR (KBr): v 3292, 3060, 1700, 1611, 1466, 1346, 1301, 1212, 1172, 1066, 997, 934, 903, 848, 752, 695, 668, $635 \mathrm{~cm}^{-1}$. HRMS: Calcd for $\mathrm{C}_{18} \mathrm{H}_{16} \mathrm{O}_{2} \mathrm{~N}_{2}[\mathrm{M}+\mathrm{Na}]^{+}$ 331.1053; found 315.1064.

\subsection{General Procedure for the Synthesis of Unsymmetrical 3,3Di(indolyl)indolin-2-ones (1e to 5e)}

A mixture of 3-indolyl-3-hydroxy oxindoles $(0.3400 \mathrm{mmol}, 100 \mathrm{mg})$ and indole $(0.4100 \mathrm{mmol}, 48 \mathrm{mg})$ was added to a microwave-oven reaction vial, and then irradiated for 10 minutes at $150^{\circ} \mathrm{C}$. Then the residue as washed with $2 \mathrm{~mL}$ of EtOAc and mixed with silicagel and then evaporated the solvent under reduced pressure, 
and the mixture was purified by column chromatography using EtOAc in hexanes.

\subsubsection{3-(5-Methoxy-1H-indol-3-yl)-3-(1H-indol-3-yl)indolin-2-one (1e)}

White solid; mp: $280^{\circ} \mathrm{C}-282^{\circ} \mathrm{C} .{ }^{1} \mathrm{H}$ NMR $\left(400 \mathrm{MHz}\right.$, DMSO- $\left.d_{6}\right): \delta 10.95$ (s, $\left.1 \mathrm{H}\right), 10.79(\mathrm{~s}, 1 \mathrm{H}), 10.59(\mathrm{~s}, 1 \mathrm{H})$, $7.35(\mathrm{~d}, J=8 \mathrm{~Hz}, 1 \mathrm{H}), 7.25-7.22(\mathrm{~m}, 4 \mathrm{H}), 7.03-6.98(\mathrm{~m}, 2 \mathrm{H}), 6.93(\mathrm{t}, J=7.2 \mathrm{~Hz}, 1 \mathrm{H}), 6.89-6.81(\mathrm{~m}, 3 \mathrm{H})$, 6.70 - 6.67 (m, 2H), 3.51 (s, 3H). ${ }^{13} \mathrm{C}$ NMR (100 MHz, DMSO-d $\left.\mathrm{d}_{6}\right): \delta 178.7,152.4,141.3,136.9,134.5,132.1$, 127.8, 126.0, 125.7, 125.0, 124.9, 124.3, 124.2, 121.4, 120.9, 120.7, 118.2, 112.0, 111.5, 110.4, 109.5, 103.3, 55.1, 52.5. IR (KBr): v 3371, 1737, 1676, 1620, 1577, 1472, 1418, 1372, 1339, 1291, 1208, 1174, 1129, 1101, 1053, 1015, 958, 930, 889, 837, 796, 746, 679, $637 \mathrm{~cm}^{-1}$. HRMS: Calcd for $\mathrm{C}_{25} \mathrm{H}_{19} \mathrm{O}_{2} \mathrm{~N}_{3}[\mathrm{M}+\mathrm{Na}]^{+} 416.1369$; found 416.1383 .

\subsubsection{3-1-Methyl(5-methoxy-1H-indol-3-yl)-3-(1H-indol-3-yl)indolin-2-one (2e)}

White solid; mp: $280^{\circ} \mathrm{C}-282^{\circ} \mathrm{C} .{ }^{1} \mathrm{H}$ NMR $\left(400 \mathrm{MHz}, \mathrm{DMSO}-\mathrm{d}_{6}\right): \delta 10.97$ (s, $\left.1 \mathrm{H}\right), 10.82(\mathrm{~s}, 1 \mathrm{H}), 7.37$ - 7.32 (m, 2H), 7.28 - $7.23(\mathrm{~m}, 2 \mathrm{H}), 7.19$ - $7.16(\mathrm{~m}, 2 \mathrm{H}), 7.04-7.00(\mathrm{~m}, 2 \mathrm{H}), 6.89$ (d, $J=2.4 \mathrm{~Hz}, 1 \mathrm{H}), 6.82$ - 6.77 (m, 2H), 6.69 (dd, $J=2.4 \mathrm{~Hz}, J=8.8 \mathrm{~Hz}, 1 \mathrm{H}), 6.58(\mathrm{~d}, J=2.4 \mathrm{~Hz}, 1 \mathrm{H}), 3.50$ (s, 3H), 3.26 (s, $3 \mathrm{H}) .{ }^{13} \mathrm{C}$ NMR $(100 \mathrm{MHz}$, DMSO-d $\left.{ }_{6}\right): \delta 177.0,152.5,142.8,137.0,133.7,132.2,128.1,126.0,125.7,125.1,124.7,124.5,122.3,121.0$, 120.7, 118.4, 113.9, 113.6, 112.2, 111.7, 110.6, 108.6, 103.0, 55.1, 52.2, 26.3. IR (KBr): v 3396, 3345, 3314, 2924, 2854, 2363, 2337, 1734, 1692, 1608, 1462, 1423, 1350, 1292, 1212, 1172, 1123, 1086, 1038, 1016, 913, 891, 844, 795, 743, 692, $650 \mathrm{~cm}^{-1}$. HRMS: Calcd for $\mathrm{C}_{26} \mathrm{H}_{21} \mathrm{O}_{2} \mathrm{~N}_{3}[\mathrm{M}+\mathrm{Na}]^{+} 430.1531$; found 430.1538 .

\subsubsection{3-1-Allyl(5-methoxy-1H-indol-3-yl)-3-(1H-indol-3-yl)indolin-2-one (3e)}

White solid; mp: 266 - 268. ${ }^{1} \mathrm{H}$ NMR (400 MHz, DMSO- $d_{6}$ ): $\delta 10.99$ (s, $\left.1 \mathrm{H}\right), 10.83$ (s, $1 \mathrm{H}$ ), 7.36 (d, $J=8.4 \mathrm{~Hz}$, $1 \mathrm{H}), 7.33-7.29$ (m, 2H), 7.24 (d, $J=8.4 \mathrm{~Hz}, 1 \mathrm{H}), 7.20$ (d, $J=8 \mathrm{~Hz}, 1 \mathrm{H}), 7.11(\mathrm{~d}, J=7.6 \mathrm{~Hz}, 1 \mathrm{H}), 7.02(\mathrm{t}, J=$ $7.6 \mathrm{~Hz}, J=14.8 \mathrm{~Hz}, 2 \mathrm{H}), 6.89$ (d, $J=2.4 \mathrm{~Hz}, 1 \mathrm{H}), 6.82(\mathrm{~d}, J=2.8 \mathrm{~Hz}, 1 \mathrm{H}), 6.79(\mathrm{~d}, J=7.2 \mathrm{~Hz}, 1 \mathrm{H}), 6.68$ (dd, $J$ $=2.4 \mathrm{~Hz}, J=8.4 \mathrm{~Hz}, 1 \mathrm{H}), 6.55(\mathrm{~d}, J=2.4 \mathrm{~Hz}, 1 \mathrm{H}), 5.95-5.86(\mathrm{~m}, 1 \mathrm{H}), 5.21(\mathrm{~d}, J=1.6 \mathrm{~Hz}, 1 \mathrm{H}), 5.18-5.15(\mathrm{~m}$, $1 \mathrm{H}), 4.43$ (d, $J=4.8 \mathrm{~Hz}, 2 \mathrm{H}), 3.48$ (s, 3H). ${ }^{13} \mathrm{C}$ NMR (100 MHz, DMSO-d 6 ): $\delta$ 176.8, 152.6, 141.8, 137.0, 133.8, 132.2, 128.0, 126.0, 125.7, 125.2, 124.8, 124.4, 122.3, 121.1, 120.8, 118.4, 117.2, 114.0, 113.6, 112.3, 111.8, 110.8, 109.3, 102.9, 55.1, 52.2, 41.8. IR (KBr): v 3754, 3708, 3345, 3010, 1736, 1669, 1606, 1540, 1478, 1456, 1358, 1293, 1208, 1171, 1093, 1018, 926, 841, 798, 744, 702, $632 \mathrm{~cm}^{-1}$. HRMS: Calcd for $\mathrm{C}_{28} \mathrm{H}_{23} \mathrm{O}_{2} \mathrm{~N}_{3}[\mathrm{M}+$ $\mathrm{Na}^{+}$456.1688; found 456.1701 .

\subsubsection{3-1-Benzyl(5-methoxy-1H-indol-3-yl)-3-(1H-Indol-3-yl)indolin-2-one (4e)}

White solid; mp: $260^{\circ} \mathrm{C}-262^{\circ} \mathrm{C} .{ }^{1} \mathrm{H}$ NMR $\left(400 \mathrm{MHz}\right.$, DMSO- $\left.d_{6}\right): \delta 11.00(\mathrm{~s}, 1 \mathrm{H}), 10.9(\mathrm{~s}, 1 \mathrm{H}), 7.38-7.37$ (m, 3H), 7.32 - 7.25 (m, 6H), 7.15 - 7.09 (m, 2H), 7.04 - 6.98 (m, 2H), 6.93 (d, $J=2 \mathrm{~Hz}, 1 \mathrm{H}), 6.85$ (d, $J=2.4 \mathrm{~Hz}$, 1H), 6.75 (t, $J=7.6 \mathrm{~Hz}, J=15.2 \mathrm{~Hz}, 1 \mathrm{H}), 6.69$ (dd, $J=2 \mathrm{~Hz}, J=8.8 \mathrm{~Hz}, 1 \mathrm{H}), 6.56$ (d, $J=2 \mathrm{~Hz}, 1 \mathrm{H}), 5.02$ (s, 2H), 3.42 (s, 3H). ${ }^{13} \mathrm{C}$ NMR (100 MHz, DMSO-d 6 ): $\delta 177.2$, 152.5, 141.8, 137.0, 133.8, 132.2, 128.6, 127.9, 127.5, 126.0, 125.6, 125.2, 124.9, 124.5, 122.4, 121.1, 120.7, 118.3, 113.9, 113.4, 112.2, 111.7, 110.8, 109.3, 103.0, 55.0, 52.3, 43.0. IR (KBr): v 3347, 3025, 2850, 1699, 1676, 1607, 1535, 1483, 1458, 1358, 1291, 1209, 1172, 1133, 1099, 1016, 930, 847, 799, 745, 698, $63 \mathrm{~cm}^{-1}$. HRMS: Calcd for $\mathrm{C}_{32} \mathrm{H}_{25} \mathrm{O}_{2} \mathrm{~N}_{3}[\mathrm{M}+\mathrm{Na}]^{+} 506.1839$ found 506.1848 .

\subsubsection{3-1-(Prop-2-ynyl)(5-methoxy-1H-indol-3-yl)-3-(1H-indol-3-yl)indolin-2-one (5e)}

White solid; mp: $266^{\circ} \mathrm{C}-268^{\circ} \mathrm{C} .{ }^{1} \mathrm{H}$ NMR $\left(400 \mathrm{MHz}\right.$, DMSO- $\left.d_{6}\right): \delta 10.99(\mathrm{~s}, 1 \mathrm{H}), 10.85(\mathrm{~s}, 1 \mathrm{H}), 7.38$ - 7.36 (m, 2H), 7.27 - $7.25(\mathrm{~m}, 4 \mathrm{H}), 7.08$ - $7.01(\mathrm{~m}, 2 \mathrm{H}), 6.87(\mathrm{~m}, 1 \mathrm{H}), 6.81$ - $6.79(\mathrm{~m}, 2 \mathrm{H}), 6.71$ - $6.69(\mathrm{~m}, 1 \mathrm{H}), 6.63(\mathrm{~s}$, 1H), $4.65(\mathrm{~s}, 2 \mathrm{H}), 3.51(\mathrm{~s}, 3 \mathrm{H}), 3.31(\mathrm{~s}, 1 \mathrm{H}) .{ }^{13} \mathrm{C}$ NMR (100 MHz, DMSO-d $\left.\mathrm{d}_{6}\right): \delta 176.3,152.6,140.9,137.0$, 133.6, 132.2, 128.0, 125.9, 125.6, 125.3, 124.9, 124.6, 122.7, 121.2, 120.9, 118.4, 113.8, 113.2, 112.3, 111.7, 111.0, 109.4, 103.0, 78.2, 74.5, 55.2, 52.3, 29.0. IR (KBr): v 3388, 3326, 3267, 1689, 1605, 1580, 1482, 1460, 1427, 1378, 1356, 1338, 1295, 1250, 1209, 1173, 1128, 1100, 1037, 1013, 932, 911, 888, 857, 800, 748, 703, $660 \mathrm{~cm}^{-1}$. HRMS: Calcd for $\mathrm{C}_{28} \mathrm{H}_{21} \mathrm{O}_{2} \mathrm{~N}_{3}[\mathrm{M}+\mathrm{Na}]^{+}$454.1531; found 454.1538 .

\section{Conclusion}

A simple and green alternative protocol for the synthesis 3-indolyl-3-hydroxy oxindoles in moderate to excellent yields is reported here. The unsymmetrical 3,3-di(indolyl)indolin-2-ones are also obtained in moderate to good 
yields under microwave irradiation. The highlights of the method are that no solvent, no catalysts are needed, and the reaction times are very short, i.e. five minutes for 3-indolyl-3-hydroxy oxindoles and ten minutes for unsymmetrical 3,3-di(indolyl)indolin-2-ones. Hence, this methodology can be conveniently used to synthesize the hybrid molecules of isatin and indoles in a short reaction time.

\section{Acknowledgements}

We thank the Department of Biotechnology and IIT-Madras for infrastructure.

\section{References}

[1] Paira, P., Hazra, A., Kumar, S., Paira, R., Sahu, K.B., Naskar, S., Saha, P., Mondal, S., Maity, A., Banerjee, S. and Mondal, N.B. (2009) Efficient Synthesis of 3,3-Diheteroaromatic Oxindole Analogues and Their in Vitro Evaluation for Spermicidal Potential. Bioorganic \& Medicinal Chemistry Letters, 19, 4786-4789.

http://dx.doi.org/10.1016/j.bmcl.2009.06.049

[2] Subba Reddy, B.V., Rajeswari, N., Sarangapani, M., Prashanthi, Y., Ganji, R.J. and Addlagatta, A. (2012) Iodine-Catalyzed Condensation of Isatin with Indoles: A Facile Synthesis of Di(indolyl)indolin-2-ones and Evaluation of Their Cytotoxicity. Bioorganic \& Medicinal Chemistry Letters, 22, 2460-2463. http://dx.doi.org/10.1016/j.bmcl.2012.02.011

[3] Pajouhesh, H., Parson, R. and Popp, F.D. (1983) Potential Anticonvulsants VI: Condensation of Isatins with Cyclohexanone and Other Cyclic Ketones. Journal of Pharmaceutical Sciences, 72, 318-321. http://dx.doi.org/10.1002/jps.2600720330

[4] DeLorbe, J.E., Jabri, S.Y., Mennen, S.M., Overman, L.E. and Zhang, F.L. (2011) Enantioselective Total Synthesis of (+)-Gliocladine C: Convergent Construction of Cyclotryptamine-Fused Polyoxopiperazines and a General Approach for Preparing Epidithiodioxopiperazines from Trioxopiperazine Precursors. Journal of the American Chemical Society, 133, 6549-6552. http://dx.doi.org/10.1021/ja201789v

[5] Guo, C., Song, J., Huang, J.-Z., Chen, P.-H., Luo, S.-W. and Gong, L.-Z. (2012) Core-Structure-Oriented Asymmetric Organocatalytic Substitution of 3-Hydroxyoxindoles: Application in the Enantioselective Total Synthesis of (+)-Folicanthine. Angewandte Chemie-International Edition, 51, 1046-1050. http://dx.doi.org/10.1002/anie.201107079

[6] Berens, U., Brown, J.M., Long, J. and Selke, R.D. (1996) Synthesis and Resolution of 2,2'-Bis-diphenylphosphino [3,3']biindolyl, a New Atropisomeric Ligand for Transition Metal Catalysis. Tetrahedron: Asymmetry, 7, $285-292$. http://dx.doi.org/10.1016/0957-4166(95)00447-5

[7] Kumar, V.P., Reddy, V.P., Sridhar, R., Srinivas, B., Narender, M. and Rao, K.R. (2008) Supramolecular Synthesis of 3-Indolyl-3-hydroxy Oxindoles under Neutral Conditions in Water. Journal of Organic Chemistry, 73, 1646-1648. http://dx.doi.org/10.1021/jo702496s

[8] Jafarpour, M., Rezaeifard, A., Gazkar, S. and Danehchin, M. (2011) Catalytic Activity of a Zirconium (IV) Schiff Base Complex in Facile and Highly Efficient Synthesis of Indole Derivatives. Transition Metal Chemistry, 36, 685-690. http://dx.doi.org/10.1007/s11243-011-9519-6

[9] Sarrafi, Y., Alimohammadi, K., Sadatshahabi, M. and Norozipoor, N. (2012) An Improved Catalytic Method for the Synthesis of 3,3-Di(indolyl)oxindoles Using Amberlyst 15 as a Heterogeneous and Reusable Catalyst in Water. Monatshefte für Chemie, 143, 1519-1522. http://dx.doi.org/10.1007/s00706-012-0723-7

[10] Jafarpour, M., Rezaeifard, A. and Gorzin, G. (2011) Enhanced Catalytic Activity of Zr(IV) Complex with Simple Tetradentate Schiff Base Ligand in the Clean Synthesis of Indole Derivatives. Inorganic Chemistry Communications, 14, 1732-1736. http://dx.doi.org/10.1016/j.inoche.2011.07.017

[11] Kamal, A., Srikanth, Y.V.V., Khan, M.N.A., Shaik, T.B. and Ashraf, M. (2010) Synthesis of 3,3-Diindolyl Oxyindoles Efficiently Catalysed by $\mathrm{FeCl}_{3}$ and Their in Vitro Evaluation for Anticancer Activity. Bioorganic \& Medicinal Chemistry Letters, 20, 5229-5231. http://dx.doi.org/10.1016/j.bmcl.2010.06.152

[12] Azizian, J., Mohammadi, A.A., Karimi, N., Mohammadizadeh, M.R. and Karimi, A.R. (2006) Silica Sulfuric Acid a Novel and Heterogeneous Catalyst for the Synthesis of Some New Oxindole Derivatives. Catalysis Communications, 7, 752-755. http://dx.doi.org/10.1016/j.catcom.2006.01.026

[13] Alinezhad, H., Haghighi, A.H. and Salehian, F. (2010) A Green Method for the Synthesis of Bis-Indolylmethanes and 3,3'-Indolyloxindole Derivatives Using Cellulose Sulfuric Acid under Solvent-Free Conditions. Chinese Chemical Letters, 21, 183-186. http://dx.doi.org/10.1016/j.cclet.2009.09.001

[14] Saffar-Teluri, A. (2014) Boron Trifluoride Supported on $\mathrm{Nano}_{-} \mathrm{SiO}_{2}$ : An Efficient and Reusable Heterogeneous Catalyst for the Synthesis of Bis(indolyl)methanes and Oxindole Derivatives. Research on Chemical Intermediates, 40, 1061-1067. http://dx.doi.org/10.1007/s11164-013-1021-7 
[15] Sarrafi, Y., Alimohammadi, K., Sadatshahabi, M. and Norozipoor, N. (2012) An Improved Catalytic Method for the Synthesis of 3,3-Di(indolyl)oxindoles Using Amberlyst 15 as a Heterogeneous and Reusable Catalyst in Water. Monatshefte für Chemie-Chemical Monthly, 143, 1519-1522. http://dx.doi.org/10.1007/s00706-012-0723-7

[16] Karimi1, N., Oskooi1, H., Heravi, M., Saeedi, M., Zakeri, M. and Tavakoli, N. (2011) On Water: Bronsted Acidic Ionic Liquid $\left[\left(\mathrm{CH}_{2}\right)_{4} \mathrm{SO}_{3} \mathrm{HMIM}\right]\left[\mathrm{HSO}_{4}\right]$ Catalysed Synthesis of Oxindoles Derivatives. Chinese Journal of Chemistry, 29, 321-323. http://dx.doi.org/10.1002/cjoc.201190085

[17] Azizian, J., Mohammadi, A.A., Karimi, A.R. and Mohammadizadeh, M.R. (2004) $\mathrm{KAl}\left(\mathrm{SO}_{4}\right)_{2} \cdot 12 \mathrm{H}_{2} \mathrm{O}$ as a Recyclable Lewis Acid Catalyst for Synthesis of Some New Oxindoles in Aqueous Media. Journal of Chemical Research, 2004, 424-426. http://dx.doi.org/10.3184/0308234041423600

[18] Yadav, J.S., SubbaReddy, B.V., Uma, G.K., Meraj, S. and Prasad, A.R. (2006) Bismuth (III) Triflate Catalyzed Condensation of Isatin with Indoles and Pyrroles: A Facile Synthesis of 3,3-Diindolyl- and 3,3-Dipyrrolyl Oxindoles. Synthesis, 2006, 4121-4123. http://dx.doi.org/10.1055/s-2006-950373

[19] Feng, G.L., Geng, L.J. and Zhang, H.L. (2009) Facile Synthesis of 3,3-Di(indolyl)indolin-2-one Derivatives Catalyzed by $\mathrm{ZrO}_{2} / \mathrm{S}_{2} \mathrm{O}_{8}{ }^{2-}$ Solid Superacid under Grinding Condition. Chemical Journal on Internet, 11, Article ID: 111001pe.

[20] Chakrabarty, M., Sarkar, S. and Harigaya, Y. (2005) A Facile Clay-Mediated Synthesis of 3,3-Diindolyl-2-indolinones from Isatins. Journal of Chemical Research, 8, 540-542. http://dx.doi.org/10.3184/030823405774663264

[21] Deb, M.L. and Bhuyan, P.J. (2009) Water-Promoted Synthesis of 3,3'-Di(indolyl)oxindoles. Synthetic Communications, 39, 2240-2243. http://dx.doi.org/10.1080/00397910802654690

[22] Praveen, C., Ayyanar, A. and Perumal, P.T. (2011) Practical Synthesis, Anticonvulsant, and Antimicrobial Activity of N-Allyl and N-Propargyl Di(indolyl)indolin-2-ones. Bioorganic \& Medicinal Chemistry Letters, 21, 4072-4077. http://dx.doi.org/10.1016/j.bmcl.2011.04.117

[23] Wang, S.Y. and Ji, S.J. (2006) Facile Synthesis of 3,3-Di(heteroaryl)indolin-2-one Derivatives Catalyzed by Ceric Ammonium Nitrate (CAN) under Ultrasound Irradiation. Tetrahedron, 62, 1527-1535. http://dx.doi.org/10.1016/j.tet.2005.11.011

[24] Moghadam, K.R., Kiasaraie, M.S. and Amlashi, H.T. (2010) Synthesis of Symmetrical and Unsymmetrical 3,3-Di(indolyl)indolin-2-ones under Controlled Catalysis of Ionic Liquids. Tetrahedron, 66, 2316-2321. http://dx.doi.org/10.1016/j.tet.2010.02.017

[25] Nikpassand, M., Mamaghani, M., Tabatabaeian, K. and Samimi, H.A. (2010) An Efficient and Clean Synthesis of Symmetrical and Unsymmetrical 3,3-Di(indolyl)indolin-2-ones Using KSF. Synthetic Communications, 40, 3552-3560. http://dx.doi.org/10.1080/00397910903457399

[26] Shanthi, G., Lakshmi, N.V. and Perumal, P.T. (2009) A Simple and Eco-Friendly Synthesis of 3-Indolyl-3-hydroxy Oxindoles and 11-Indolyl-11H-indeno[1,2-b]quinoxalin-11-ols in Aqueous Media. ARKIVOC, 2009, 121-130. http://dx.doi.org/10.3998/ark.5550190.0010.a12

[27] Meshram, H.M., Kumar, D.A., Goud, P.R. and Reddy, B.C. (2010) ChemInform Abstract: Triton B Assisted, Efficient, and Convenient Synthesis of 3-Indolyl-3-hydroxy Oxindoles in Aqueous Medium. Synthetic Communications, 40, 3945. http://dx.doi.org/10.1002/chin.201025093

[28] Hosseini-Sarvari, M. and Tavakolian, M. (2012) Preparation, Characterization, and Catalysis Application of Nano-Rods Zinc Oxide in the Synthesis of 3-Indolyl-3-hydroxy Oxindoles in Water. Applied Catalysis A: General, 441-442, 65-71. http://dx.doi.org/10.1016/j.apcata.2012.07.009

[29] Khorshidi, A. and Tabatabaeian, K.J. (2011) An Ultrasound-Promoted Green Approach for the Synthesis of 3-(Indol3-yl)-3-hydroxyindolin-2-ones Catalyzed by Fe(III). Journal of the Serbian Chemical Society, 76, 1347-1353. http://dx.doi.org/10.2298/JSC110420120K

[30] Makarem, S., Fakhari, A.R. and Mohammadi, A.A. (2012) Electro-Organic Synthesis of Nanosized Particles of 3-Hydroxy3-(1H-indol-3-yl)indolin-2-one Derivatives. Monatshefte für Chemie-Chemical Monthly, 143, 1157-1160. http://dx.doi.org/10.1007/s00706-011-0693-1

[31] Srihari, G. and Murthy, M.M. (2011) Kaolin/KOH Is an Efficient Heterogeneous Catalyst for the Synthesis of 3-Hydroxy3-indolyl Oxindoles. Synthetic Communications, 41, 2684-2692. http://dx.doi.org/10.1080/00397911.2010.515342

[32] Deng, J., Zhang, S., Ding, P., Jiang, H., Wang, W. and Li, J. (2010) Facile Creation of 3-Indolyl-3-hydroxy-2-oxindoles by an Organocatalytic Enantioselective Friedel-Crafts Reaction of Indoles with Isatins. Advanced Synthesis \& Catalysis, 352, 833-838. http://dx.doi.org/10.1002/adsc.200900851

[33] Hanhan, N.V., Sahin, A.H., Chang, T.W., Fettinger, J.C. and Franz, A.K. (2010) Catalytic Asymmetric Synthesis of Substituted 3-Hydroxy-2-oxindoles. Angewandte Chemie International Edition, 49, 744-747.

http://dx.doi.org/10.1002/anie.200904393 
[34] Prathima, P.S., Rajesh, P., Rao, J.V., Kailash, U.S., Sridhar, B. and Rao, M.M. (2014) “On Water” Expedient Synthesis of 3-Indolyl-3-hydroxy Oxindole Derivatives and Their Anticancer Activity in Vitro. European Journal of Medicinal Chemistry, 84, 155-159. http://dx.doi.org/10.1016/j.ejmech.2014.07.004

[35] Lakhdar, S., Westermaier, M., Terrier, F., Goumont, R., Boubaker, T., Ofial, A.R. and Mayr, H. (2006) Nucleophilic Reactivities of Indoles. The Journal of Organic Chemistry, 71, 9088-9095. http://dx.doi.org/10.1021/jo0614339 